\title{
0449. Surface EMG of extra-diaphragmatic muscles can assess muscle unloading during neurally adjusted ventilatory support
}

\author{
P Somhorst ${ }^{1,2^{*}}$, M van Mourik ${ }^{1,2}$, AA Koopman ${ }^{1,2}$, D Gommers $^{1}$ \\ From ESICM LIVES 2014 \\ Barcelona, Spain. 27 September - 1 October 2014
}

\section{Introduction}

Neurally adjusted ventilatory assist (NAVA) is used to adapt mechanical ventilation to patient demand while unloading respiratory muscles. Titration methods are focused on sustained unloading of the diaphragm while maintaining a stable tidal volume [1]. It is hypothesized that the activity of accessory respiratory muscles can supply extra information about muscle unloading and patient comfort during NAVA ventilation.

\section{Objectives}

To assess the extra-diaphragmatic muscle activity (EDMA) during $100 \%, 50 \%$ and $150 \%$ of titrated NAVA level.

\section{Methods}

EDMA was measured in ventilated patients with mild ARDS. EDMA was defined as the amplitude of the combined surface EMG at the scalene and sternomastoid muscle (Dipha16, InBiolab, Groningen, The Netherlands). A baseline NAVA level (NAVA100) was titrated using the diaphragm activity (EAdi) response to changing NAVA levels, according to Brander et al. [1]. Patients were ventilated with NAVA100, NAVA50 (50\% of NAVA100) and NAVA150 (150\% NAVA100) for a period of 15 minutes.

\section{Results}

Twenty-one patients were included. In six patients EDMA was absent during NAVA100, so NAVA titration was sufficient to unload accessory respiratory muscles. Fifteen patients (71\%) showed EDMA during NAVA100. In seven patients (33\%) EDMA increased at NAVA50 and decreased during NAVA150. In one patient, EDMA decreased at NAVA150. One patient only showed EDMA during NAVA50. In three patients EDMA decreased at NAVA50 or increased at NAVA150. Nine patients (43\%) showed no change in EDMA after a change of NAVA level.

\section{Conclusions}

Measurement of extra-diaphragmatic muscle activity using surface EMG during NAVA ventilation might be helpful in titration of ventilatory assist level in order to optimize patient's comfort.

\section{Authors' details}

${ }^{1}$ Erasmus MC, Adult Intensive Care, Rotterdam, Netherlands. ${ }^{2}$ University of Twente, Institute of Technical Medicine, Enschede, Netherlands.

\section{Published: 26 September 2014}

\section{Reference}

1. Brander L, Leong-Poi H, Beck J, Brunet F, Hutchison SJ, et al: Titra- tion and implementation of neurally adjusted ventilatory assist in critically ill patients. Chest 2009, 135(3):695-703.

doi:10.1186/2197-425X-2-S1-012

Cite this article as: Somhorst et al:: 0449. Surface EMG of extra-

diaphragmatic muscles can assess muscle unloading during neurally adjusted ventilatory support. Intensive Care Medicine Experimental 20142 (Suppl 1):012. 